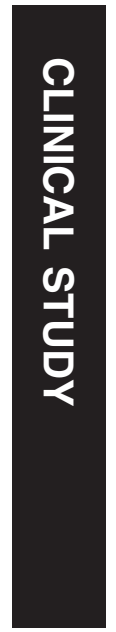

\section{Effect of senescence on ocular blood flow in the retina, neuroretinal rim and lamina cribrosa, using scanning laser Doppler flowmetry}

\begin{abstract}
Purpose To determine the effects of age on blood flow measurements obtained using the scanning laser Doppler flowmeter (SLDF). Method Using SLDF (Heidelberg retina flowmeter, Heidelberg Engineering, Germany) three $10^{\circ}$ images were taken of the superior temporal retina and three further images of the superior temporal neuroretinal rim in 15 young, healthy subjects (mean age 27.9 years \pm 6.2 years) and 15 mature, healthy subjects (mean age 65.2 years \pm 13.7 years).

In addition, measurements were taken of the lamina cribrosa in $\mathbf{1 2}$ of the volunteers from each subject group (mean age $27.1 \pm 6.3$ years and $64.8 \pm 13.2$ years respectively). Using a $10 \times 10$ pixel measurement frame, blood flow readings were obtained at a predetermined position on the retina, neuroretinal rim and lamina cribrosa. Student's two-tailed unpaired $t$-tests were used to compare measures of blood flow, volume and velocity between the two subject groups $(P<0.05)$. In addition, linear regression analysis was used to assess the relationship between age and blood flow, volume and velocity at the retina, neuroretinal rim and lamina cribrosa. Results Retinal blood volume measured at the retina was significantly lower in the mature compared with the young subject group $(P=0.01)$. Mature subjects also exhibited reduced blood flow and velocity at the neuroretinal $\operatorname{rim}(P=0.01$ for both parameters) and lamina cribrosa $(P=0.008$ and $P=0.01$ respectively). Regression analysis revealed negative trends for all blood flow parameters in each of the anatomical areas with advancing age.
\end{abstract}

${ }^{1}$ Neurosciences Research Institute

School of Life and Health Sciences

Aston University Birmingham, UK

${ }^{2}$ Department of

Ophthalmology

Birmingham Heartlands

Hospital and Solihull NHS

Trust

Birmingham, UK

Correspondence:

Dr SL Hosking

Neurosciences Research

Institute

School of Life and Health

Sciences

Aston University

Aston Triangle

Birmingham B4 7ET, UK

Tel: +44 (0)1213593611

$\times 5172$

Fax: $+44(0) 1213334220$

E-mail: s.l.hosking@

aston.ac.uk

Received: 17 January 2001 Accepted in revised form: 13 August 2001
SJ Embleton 1,2 , SL Hosking ${ }^{1,2}$, EJ Roff Hilton ${ }^{1,2}$ and IA Cunliffe ${ }^{2}$

Significant negative correlations were obtained for retinal blood volume $(r=-0.455, P<0.05)$, neuroretinal rim blood velocity $(r=-0.359, P<0.05)$ and lamina cribrosa blood volume $(r=-0.475, P<0.05)$. Conclusion Capillary blood flow in the retina, neuroretinal rim and lamina cribrosa decreases with advancing age. This may be of consequence in the progression of chronic ocular diseases such as glaucoma, and should be considered in the longitudinal determination of change in disease monitoring.
Eye (2002) 16, 156-162. DOI: 10.1038/

sj/EYE/6700100

Keywords: age; blood flow; eye; scanning laser Doppler flowmetry

\section{Introduction}

The Heidelberg retina flowmeter (HRF; Heidelberg Engineering, Germany) is a scanning laser Doppler flowmeter (SLDF) recommended for the investigation of fundus blood flow in vivo. It combines the confocal scanning laser technique with Doppler flowmetry to generate two-dimensional maps of retinal perfusion. ${ }^{1,2}$

The principles of this technique have been described in detail elsewhere. ${ }^{2}$ In brief, infrared light with a wavelength of $780 \mathrm{~nm}$ is used to scan the retina. Stationary targets, such as the blood vessel wall, and moving objects, such as the red blood cells, reflect the laser beam. The light reflected by the moving red blood cells is frequency-shifted due to the optical Doppler effect and this shift in 
frequency is proportional to the rate of movement of the corpuscles. The frequency shift itself is too small to measure directly, but after interference between this and the light reflected from stationary targets, a beat frequency is generated. The beat frequency is measured at each point by a photodiode detector in the HRF. ${ }^{2}$ Following fast Fourier transformation, the data are used to derive indices of blood flow, volume and velocity. ${ }^{3}$ The number and velocity of moving corpuscles is obtained by multiple measurements at each pixel location over a fixed period of time. The total blood flow is the product of the number and rate of corpuscles flowing past each pixel location over time.

Many investigators have reported favourably on the reproducibility and validity of measurements obtained using the HRF. ${ }^{4-7}$ Compromised ocular blood flow has been shown to underlie various ocular pathologies. Using the HRF, altered blood flow has previously been reported in diabetes, ${ }^{8}$ age-related macular degeneration $^{9}$ and glaucoma. ${ }^{1,7,10,11}$ Many of the changes associated with reduced blood flow in ocular disease are known to become more prevalent with age, which is a known risk factor in glaucoma. ${ }^{12,13}$ Blood flow deficits identified using the HRF have been reported in both normal-tension glaucoma ${ }^{14}$ and primary open angle glaucoma ${ }^{1,11}$ in the retina, neuroretinal rim and lamina cribrosa. Since blood flow is known to decrease with increasing age, ${ }^{15-17}$ this may be a confounding factor in the development or progression of glaucoma.

The optic nerve head is supplied by branches of the posterior ciliary arteries, ${ }^{18}$ with influence from the central retinal artery in the superficial layers. ${ }^{19}$ The choroid receives its nutrients from the posterior ciliary arteries. ${ }^{20}$ Over time the structure of the arteries changes, culminating in a decrease in the elasticity of blood vessels. ${ }^{21}$ In addition, the structure of endothelial cells alters with increasing age and these cells play a crucial role in vascular tone and regulation. ${ }^{22}$ One might predict, therefore, that these alterations result in reduced retinal blood flow. Reductions in blood velocity and flow with advancing age have been demonstrated using pulsed Doppler sonography and scanning laser Doppler flowmetry. ${ }^{17}$ In addition, it has been demonstrated that ocular pulsatility, derived from blood inflow during cardiac systole and influenced by, among other things, vascular elasticity, reduces with age. $^{15,16}$

The lamina cribrosa is situated in the central region of the optic nerve head. It is an elastic structure composed of perforated cribriform plates through which nerve fibres and the central retinal artery pass. The lamina cribrosa receives its blood supply from the choroidal arteries and the short posterior ciliary arteries. ${ }^{23}$ It is known that with age the components of the cribriform plates alter, ${ }^{24}$ this results in a stiffer structure with decreased mechanical compliance. ${ }^{25}$ It has been hypothesised that these changes are likely to make the ageing eye more susceptible to retinal ganglion cell axon damage, especially when coupled with fluctuations in intraocular pressure common to primary open angle glaucoma. ${ }^{25}$ The cribiform plates are in very close proximity to one another, as are the nerve fibre axons which pass through them. During changes in intraocular pressure the cribriform plates are rearranged, and in turn exert force on the nerve fibre axons. In a more rigid structure these forces are likely to have a greater detrimental effect on the nerve fibres. In addition to the known vascular changes associated with age it is possible that a decrease in the elasticity of the lamina cribrosa may have an effect on the blood flow of the vessels that supply it with nutrients. This has been postulated as a cause of optic nerve damage in glaucoma, but has not previously been investigated in normal, healthy eyes.

The purpose of this study was to determine the effect of increasing age on ocular blood flow in the retina, neuroretinal rim and lamina cribrosa.

\section{Materials and methods}

The subject sample consisted of one eye each of 15 mature and 15 young healthy volunteers. Good-quality images were obtained for the neuroretinal rim and peripapillary retina for each subject. For the images located on the lamina cribrosa, 12 of the 15 subjects provided images of sufficient quality for analysis. Details of the subject samples used for each anatomical area of interest are given in Table 1.

Young subjects were required to be between the age of 18 and 40 years, whilst the mature subjects were all over 45 years with no upper age limit. Both subject groups were required to have a visual acuity of $6 / 9$ or

Table 1 Details of the subject samples used in the study for the retina, neuroretinal rim (NRR) and lamina cribrosa

\begin{tabular}{|c|c|c|c|c|c|c|c|}
\hline \multirow[t]{2}{*}{ Area } & \multirow[t]{2}{*}{ Group } & \multicolumn{2}{|c|}{ Gender } & \multicolumn{2}{|c|}{ Eye } & \multirow{2}{*}{$\begin{array}{l}\text { Mean age } \\
\pm S D \\
\text { (years) }\end{array}$} & \multirow{2}{*}{$\begin{array}{l}\text { Age } \\
\text { range } \\
\text { (years) }\end{array}$} \\
\hline & & $F$ & $M$ & $R$ & $L$ & & \\
\hline $\begin{array}{l}\text { Retina } \\
\text { and NRR }\end{array}$ & Young & 7 & 8 & 8 & 7 & $27.8 \pm 6.2$ & $20-38$ \\
\hline $\begin{array}{l}\text { Retina } \\
\text { and NRR }\end{array}$ & Mature & 7 & 8 & 8 & 7 & $65.2 \pm 13.7$ & $7 \quad 48-82$ \\
\hline $\begin{array}{l}\text { Lamina } \\
\text { cribrosa }\end{array}$ & Young & 6 & 6 & 6 & 6 & $27.1 \pm 6.3$ & $20-38$ \\
\hline $\begin{array}{l}\text { Lamina } \\
\text { cribrosa }\end{array}$ & Mature & 7 & 5 & 6 & 6 & $64.8 \pm 13.2$ & $48-82$ \\
\hline
\end{tabular}


better in each eye with a refractive error of less than 8 dioptres mean sphere. Intraocular pressures were less than $22 \mathrm{mmHg}$ in both eyes, anterior angles were open and ocular examination confirmed a normal optic nerve head appearance. There was no history of ocular trauma or surgery, no diabetes, no systemic hypertension or hypotension and no family history of glaucoma. Furthermore, none of the subjects were receiving any systemic or ocular medications known to affect blood flow. Two subjects from each group were smokers.

Full ethical approval from the institutions involved was granted prior to commencement of the study and informed consent was obtained from every subject. All procedures conformed to the tenets of the Declaration of Helsinki.

Following pupil dilation, and a $20 \mathrm{~min}$ resting period to ensure maximal mydriasis, one eye was chosen at random for each subject and nine HRF images were acquired: three located in the superior temporal retina, three located in the superior temporal neuroretinal rim and three located in the lamina cribrosa. Three images were taken at each anatomical area in order to obtain mean values of blood flow, volume and velocity. This was done to overcome physiological variations in blood measures occurring due to the cardiac cycle. Of the subjects included in this study, three had optic nerve head vasculature that prevented positioning of the $10 \times 10$ pixel grid on the lamina cribrosa. This was due to either interference from the larger blood vessels or the absence of any visible cupping.

The superior temporal area of the retina and neuroretinal rim were chosen for image acquisition as these areas have been shown to exhibit less variability in blood flow measures when using the HRF. ${ }^{26}$ Acetate sheets were utilised to trace the retinal and neuroretinal rim vasculature for each fundus, and a blood vessel landmark, such as a vessel bifurcation, was identified to ensure that the same retinal, neuroretinal rim and lamina cribrosa locations were used for each image. Every effort was made to keep the locations constant within each subject image series. In addition, efforts were made to optimise the reproducibility of blood flow measurements acquired by obtaining separate HRF images of the different fundus locations, thus ensuring that the DC level (direct current) of the area under examination remained consistently within the range of 110 to 150 DC. ${ }^{27}$ Images were only accepted where no or very little eye movement had occurred during image acquisition, thus ensuring perfusion maps had minimal movement saccades.

Using the HRF software (version 1.02) fast Fourier transformation was used to derive perfusion images.
Blood flow, volume and velocity (arbitrary units) were determined for each image using a $10 \times 10$ pixel square grid located at a predetermined position on the retina, neuroretinal rim and lamina cribrosa for each subject.

\section{Statistical analysis}

Student's two-tailed unpaired $t$-tests were used to test for significance between the two groups for each blood flow parameter at the retina, neuroretinal rim and lamina cribrosa. A $P$-value of less than 0.05 was considered significant.

Pearson's correlation coefficient was used to investigate a linear correlation between age and blood flow parameters.

\section{Results}

Students unpaired two-tailed $t$-tests revealed a significant reduction with age in retinal blood volume $(P=0.01)$, neuroretinal rim blood flow $(P=0.02)$, neuroretinal rim blood velocity $(P=0.01)$, lamina cribrosa blood flow $(P=0.008)$ and lamina cribrosa blood velocity $(P=0.01)$ (Table 2$)$. No significant difference was found for retinal blood flow or velocity or neuroretinal rim and lamina cribrosa blood volume between the two groups, although the trend was for blood flow parameters to decrease with increasing age (Table 2).

Figure 1 shows the mean blood flow values measured at the retina, neuroretinal rim and lamina cribrosa for the two subject groups, while Figures 2 and 3 show the mean blood volume and velocity values measured at the retina, neuroretinal rim and lamina cribrosa. Figures 4 to 6 show the linear correlation $(r)$ for retinal blood volume, neuroretinal rim blood velocity and lamina cribrosa blood volume respectively. Measures of blood flow, volume and velocity at three of the anatomical areas showed a negative trend with increasing age. Significant negative correlations were obtained for retinal blood volume $(r=-0.455, P=0.01)$, neuroretinal rim blood velocity $(r=-0.359, P=0.01)$ and lamina cribrosa blood volume $(r=-0.475, P=0.008)$. No significance was found for neuroretinal rim blood flow and lamina cribrosa blood flow and velocity.

\section{Discussion}

The results from this study suggest that capillary blood flow measured at the superior temporal retina, superior temporal neuroretinal rim and at the lamina cribrosa decreases with increasing age. A comparison 

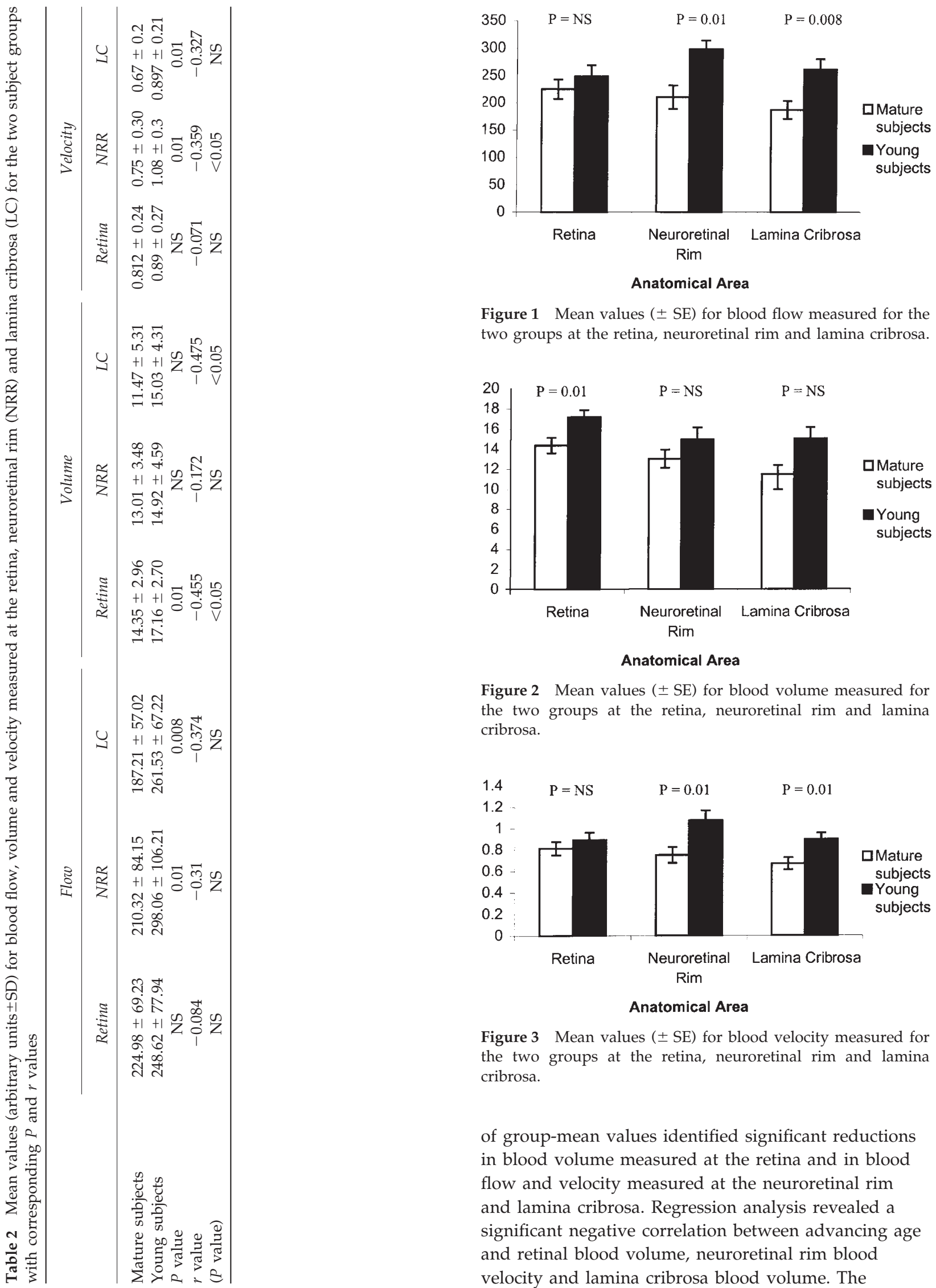

Anatomical Area

Figure 1 Mean values ( \pm SE) for blood flow measured for the two groups at the retina, neuroretinal rim and lamina cribrosa.

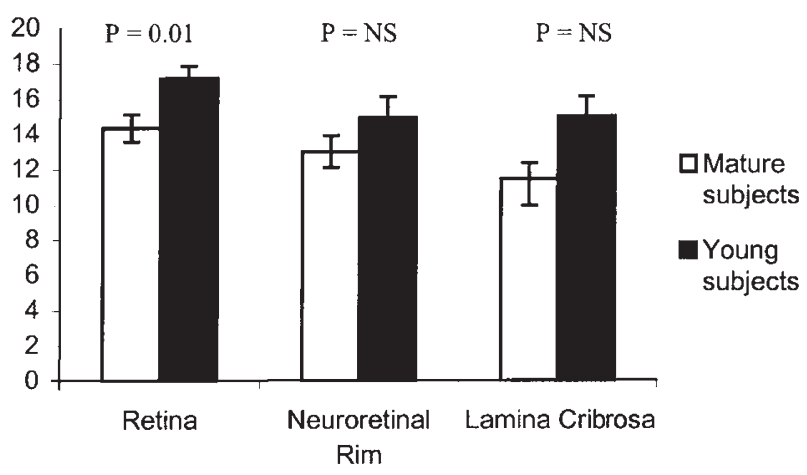

Anatomical Area

Figure 2 Mean values ( \pm SE) for blood volume measured for the two groups at the retina, neuroretinal rim and lamina cribrosa.

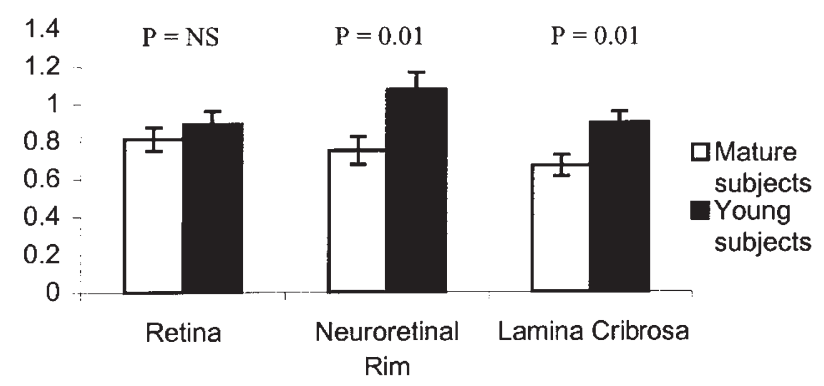

Anatomical Area

Figure 3 Mean values ( $\pm \mathrm{SE}$ ) for blood velocity measured for the two groups at the retina, neuroretinal rim and lamina cribrosa.

of group-mean values identified significant reductions in blood volume measured at the retina and in blood flow and velocity measured at the neuroretinal rim and lamina cribrosa. Regression analysis revealed a significant negative correlation between advancing age and retinal blood volume, neuroretinal rim blood velocity and lamina cribrosa blood volume. The 


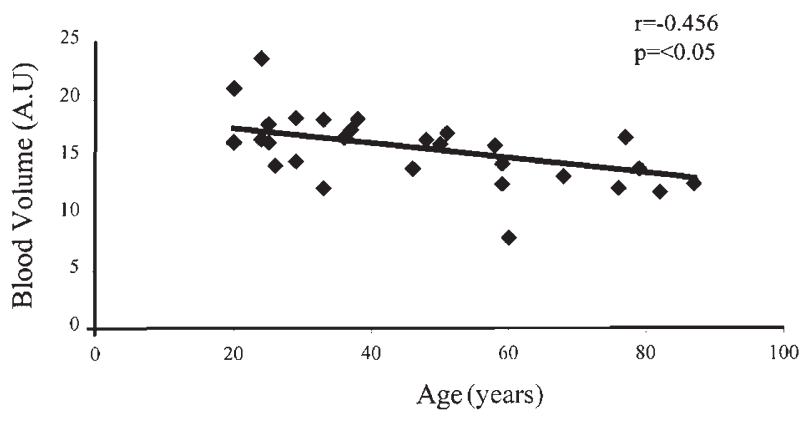

Figure 4 Effect of age on retinal blood volume.

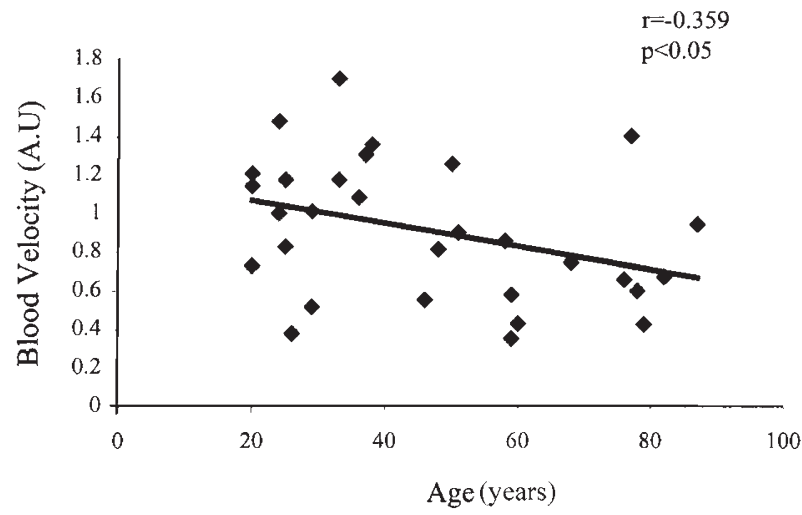

Figure 5 Effect of age on neuroretinal rim blood velocity.

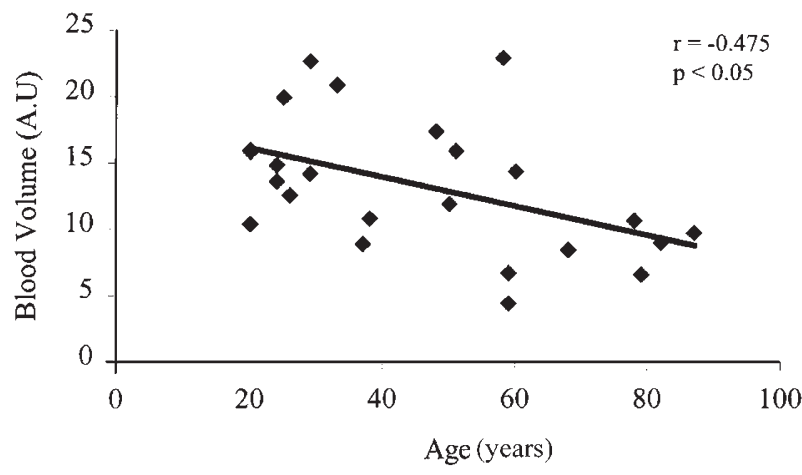

Figure 6 Effect of age on lamina cribrosa blood volume.

absence of any significant change in blood flow and velocity at the lamina cribrosa by regression analysis may be the result of the slightly smaller sample size in this group. The overall finding in this study that ocular blood flow decreases with advancing age is in agreement with previous investigations. ${ }^{15,17,28,29}$

It is known that the lamina cribrosa changes throughout life to become a stiffer, less elastic structure ${ }^{25}$ and our results revealed, for the first time, that a significant reduction in blood flow and velocity occurs with age in the lamina cribrosa. This supports the theory that, with age, blood flow in the choroidal arteries and short posterior ciliary arteries that supply the lamina cribrosa, decreases. This observed reduction in blood flow at the lamina cribrosa may be due to the combined effects of reduced vascular and mechanical compliance with advancing age. Furthermore, it is known that with the onset of some ocular diseases the rigidity of the lamina cribrosa increases,,$^{30}$ thus potentially contributing further to reductions in flow. With reference to glaucoma, reduced ocular blood flow has been directly implicated as a contributing factor in its pathogenesis. Nicolela et al ${ }^{11}$ found that in glaucoma patients the blood flow, volume and velocity in the lamina cribrosa were significantly lower when compared with age-matched normals.

Groh $e t a l^{17}$ investigated the effect of age on the microcirculation of the retina and neuroretinal rim using the HRF. Consistent with our findings, blood flow measured at the retina significantly reduced with advancing age; however, unlike the results from our study, the blood flow of the neuroretinal rim was not found to be significantly influenced by age. This discrepancy in results may be due to differences in the average ages of the subject groups used in each of the studies. Another contributing factor could be the DC level at which the perfusion images were obtained; this was controlled for in our study but average DC values may have been much lower in the study of Groh $e a^{17}$ as blood flow values for the retina and optic nerve head were acquired from the same image. Groh $\mathrm{et}_{\mathrm{al}}{ }^{17}$ also investigated the macrocirculation of the central retinal artery, using pulsed Doppler sonography, and reported that significant decreases in blood velocity occur with advancing age. This finding is supported by other investigators, ${ }^{31,32}$ who have found reduced retrobulbar and choroidal blood velocity with advancing age

Anatomically, the optic nerve head is supplied by both the posterior ciliary arteries and the central retinal artery (in the more superficial layers), whereas the choroid is supplied solely by the posterior ciliary arteries. If blood flow is reduced in the central retinal artery, as Groh et al ${ }^{17}$ suggest, then one would expect this to have implications for both retinal and neuroretinal rim perfusion. The observed decrease in both neuroretinal rim and retinal blood flow in this study serves to support this. Furthermore, the finding that blood flow is reduced in the lamina cribrosa suggests the presence of decreased blood flow in the posterior ciliary arteries. Alternatively, the reduction in microvascular flow in the lamina cribrosa may be restricted to the capillaries. A reduction in posterior ciliary blood flow may also have implications for choroidal perfusion. Ravalico et $a l^{15}$ reported a reduction in the pulsatile component of ocular blood 
flow with increasing age. It is known that pulsatile ocular blood flow is primarily choroidal in origin, ${ }^{33}$ thus providing indirect support for reduced choroidal flow. Further studies are required to determine the involvement of the posterior ciliary arteries in the perfusion of the ageing eye.

To summarise, age has a significant effect on neuroretinal rim and lamina cribrosa blood flow and velocity and retinal volume. Morphological changes associated with age, such as reductions in retinal ganglion cells and their axons, have previously been reported..$^{34-36}$ This depletion in nerve fibre numbers may result in a reduced requirement for ocular perfusion, or alternatively reduced perfusion may itself result in the depletion of retinal ganglion cell axons. Blood flow measurements may fall due to increases in vascular resistance following changes in the capillary vessel structure with age. ${ }^{37}$ It is known that with age the incidence of atherosclerosis increases, which reduces arterial distensibility. ${ }^{21}$ It therefore follows that with advancing age the compliance of the retrobulbar arteries, including the posterior ciliary arteries and the central retinal artery, are likely to diminish and in turn will result in a decrease in the retinal and neuroretinal rim microcirculation. Alternatively, the blood flow reductions observed may be due to loss of autoregulatory processes ${ }^{38}$ that are strongly influenced by the endothelium, and known to alter with increasing age. ${ }^{22}$ While the exact basis for reduced blood flow is uncertain, what remains clear is that advancing age results in lower ocular blood flow. It is likely that multiple factors are involved in the process of blood flow diminution with age, and such reduction may be of significance in the aetiology of some agerelated eye diseases, or indeed exacerbated by them.

\section{References}

1 Michelson G, Langhans M, Groh J. Perfusion of the juxtapapillary retina and the neuroretinal rim area in primary open angle glaucoma. J Glaucoma 1996; 5: 91-98.

2 Michelson G, Schmauss B. Two dimensional mapping of the perfusion of the retina and optic nerve head. $\mathrm{Br} J$ Ophthalmol 1995; 79: 1126-1132.

3 Bonner R, Nossal R. Model for laser Doppler measurements of blood flow in tissue. Appl Optics 1981; 20: 2097-2107.

4 Chauhan B, Smith F. Confocal scanning laser Doppler flowmetry: experiments in a model flow system. $J$ Glaucoma 1997; 6: 237-245.

5 Strenn $\mathrm{K}$ et al. Reproducibility and sensitivity of scanning laser Doppler flowmetry during graded changes in $\mathrm{PO}_{2}$. Br J Ophthalmol 1997; 81: 360-364.

6 Michelson G et al. Principle, validity, and reliability of scanning laser Doppler flowmetry. J Glaucoma 1996; 5: 99105

7 Nicolela $\mathrm{M}$ et al. Reproducibility of retinal and optic nerve head blood flow measurements with scanning laser Doppler flowmetry. J Glaucoma 1997; 6: 157-164.

8 Findl $\mathrm{O}$ et al. Ocular haemodynamics and colour contrast sensitivity in patients with type 1 diabetes. $\mathrm{Br} J$ Ophthalmol 2000; 84: 493-498.

9 Remsch H, Lang GE, Lang GK. Changes of capillary blood flow in age related macular degeneration. Invest Ophthalmol Vis Sci 1999; 40: S486.

10 Holló $\mathrm{G}$ et al. Evaluation of the peripapillary circulation in healthy and glaucoma eyes with scanning laser Doppler flowmetry. Int Ophthalmol 1997; 20: 71-77.

11 Nicolela M, Hnik P, Drance S. Scanning laser Doppler flowmeter study of retinal and optic disk blood flow in glaucomatous patients. Am J Ophthalmol 1996; 122: 775783.

12 Hayreh S. The role of age and cardiovascular disease in glaucomatous optic neuropathy. Surv Ophthalmol 1999; 43 S27-S42.

13 Garway-Heath D, Wollstein G, Hitchings R. Aging changes of the optic nerve head in relation to open angle glaucoma. Br J Ophthalmol 1997; 81: 840-845.

14 Chung $\mathrm{H}$ et al. Peripapillary retinal blood flow in normal tension glaucoma. Br J Ophthalmol 1999; 83: 466-469.

15 Ravalico G et al. Age related ocular blood flow changes. Invest Ophthalmol Visual Sci 1996; 37: 2645-2650.

16 Massey A, Geyer O, Silver DM. The effect of eye volume and age on pulsatile ocular blood flow. Invest Opthalmol Vis Sci 1999; 40: S490.

17 Groh $\mathrm{M}$ et al. Influence of age on retinal and optic nerve head circulation. Ophthalmology 1996; 103: 529-534.

18 Neetens A. Vascular supply of the optic nerve head. Neuroophthalmology 1994; 14: 113-120.

19 Buchi ER. The blood supply to the optic nerve head. In: Kaiser HJ, Flammer J, Hendrickson P (eds). Ocular Blood Flow: New Insights into the Pathogenesis of Ocular Diseases. Karger: Basel, 1996, pp 1-8.

20 Bill A. Ocular circulation. In: Moses RA (ed). Adlers Physiology of the Eye. Mosby: St Louis, 1981, pp 184-203.

21 Wadsworth RM. Calcium and vascular reactivity in ageing and hypertension. J Hypertens 1990; 8: 975-983.

22 Wei JY. Age and the cardiovascular system. N Engl J Med 1992; 327: 1735-1739.

23 Hayreh SS. Pathogenosis of optic nerve damage and visual field defects. In: Heilman LK, Richardson KT, (eds). Glaucoma: Conceptions of a Disease. Georg Thieme: Stuttgart, 1978, pp 104-107.

24 Albon J, et al. Changes in the collagenous matrix of the ageing human lamina cribosa. Br J Ophthalmol 1995; 79: 368-375.

25 Albon J, et al. Age related compliance of the lamina cribosa in human eyes. Br J Ophthalmol 2000; 84: 318-323.

26 Bohdanecka $\mathrm{Z}$ et al. Influence of acquisition parameters on hemodynamic measurements with the Heidelberg retinal flowmeter at the optic disc. J Glaucoma 1998; 7: 151-157.

27 Hosking SL et al. Detector sensitivity influences blood flow sampling in scanning laser Doppler flowmetry. Graefes Archiv Clin Exp Ophthalmol 2001; 239: 407-410.

28 Dallinger $\mathrm{S}$, et al. Age dependence of choroidal blood flow. J Am Geriatr Soc 1998; 46: 484-487.

29 Grunwald JE, Hariprasad SM, DuPont J. Effects of aging on foveolar choroidal circulation. Arch Ophthalmol 1998; 116: $150-154$.

30 Zeimer RC. Circadian variations in intraocular pressure. 
In: Ritch R, Shields MB, Krupin T (eds). The Glaucomas. CV Mosby: St Louis, 1989.

31 Harris A et al. Aging affects the retrobulbar circulation differently in women and men. Arch Ophthalmol 2000; 118: 1076-1080.

32 Straubhaar $\mathrm{M}$ et al. Chroidal laser Doppler flowmetry in healthy subjects. Arch Ophthalmol 2000; 118: 211-215.

33 James C. POBF [editorial]. Br J Ophthalmol 1998; 82: 720 721.

34 Jonas JB, Nguyen NX, Naumann GO. The retinal nerve fiber layer in normal eyes. Ophthalmology 1989; 96: 627632
35 Jonas JB, et al. Human optic nerve fiber count and optic disc size. Invest Ophthalmol Vis Sci 1992; 33: 2012-2018.

36 Gao H, Hollyfield JG. Aging of the human retina: differential loss at neurons and retinal pigment epithelial cells. Invest Ophthalmol Vis Sci 1992; 33: 1-17.

37 Lee WR, Blass GE, Shaw DC. Age-related retinal vasculopathy. Eye 1987; 1: 296-303.

38 Hayreh SS, Bill A, Sperber GO. Effects of high intraocular pressure on the glucose metabolism in the retina and optic nerve in bold atherosclerotic monkeys. Graefes Arch Clin Exp Ophthalmol 1994; 232: 745-752. 